\title{
Whole liver deceased donor liver transplantation for pediatric recipients: single-center experience for 20 years
}

\author{
Sung-Min Kim', Shin Hwang ${ }^{1}$, Jung-Man Namgung ${ }^{1}$, Dae-Yeon Kim¹ ${ }^{1}$ Tae-Yong Ha ${ }^{1}$, Gi-Won Song ${ }^{1}$, Dong-Hwan Jung ${ }^{1}$, \\ Gil-Chun Park' ${ }^{1}$ Kyung Mo Kim², Seak Hee Oh² \\ ${ }^{1}$ Department of Surgery, Asan Medical Center, University of Ulsan College of Medicine, Seoul, Korea \\ ${ }^{2}$ Department of Pediatrics, Asan Medical Center, University of Ulsan College of Medicine, Seoul, Korea
}

Background: We investigated the incidence and outcomes of pediatric deceased donor liver transplantation (DDLT) using whole liver grafts in a high-volume liver transplantation (LT) center.

Methods: The study was a retrospective single-center analysis of whole LT in pediatric recipients. The study period was set as 20 years between January 2000 and December 2019. We defined pediatric recipients and donors to be aged $\leq 18$ years.

Results: During the study period, there were 98 cases of pediatric DDLT, and 34 patients (34.7\%) received whole liver grafts. The age range of the deceased donors was 3 months to 56 years and that of pediatric recipients was 7 months to 17 years. Common primary diseases for LT were biliary atresia in 13 , acute liver failure in four, Wilson disease in four, congenital portal vein agenesis in three, and genetic metabolic diseases in three. Pediatric-to-pediatric and adult-to-pediatric whole LTs were $22(64.7 \%)$ and 12 $(35.3 \%)$, respectively. A good correlation was noted between the donor and the recipient's body weight, and the recipient's body weight and allograft's weight. Graft and overall patient survival rates were $91.2 \%$ and $91.2 \%$ at 1 year, $88.0 \%$ and $88.0 \%$ at 3 years, and $88.0 \%$ and $88.0 \%$ at 5 years, respectively.

Conclusions: The results of this study revealed that Korean Network for Organ Sharing (KONOS) regulations with donor-recipient body weight matching exhibited good performance. Considering the reciprocal trades of liver organs among pediatric and adult donors and recipients, it is necessary to establish a policy for pediatric donor liver grafts to pediatric recipients on a priority basis.

Corresponding author: Shin Hwang

E-mail: shwang@amc.seoul.kr

(c) The Korean Society for Transplantation

This is an Open Access article distributed under the terms of the Creative Commons Attribution Non-Commercial License (http://creativecommons.org/licenses/by-nc/4.0/) which permits unrestricted non-commercial use, distribution, and reproduction in any medium, provided the original work is properly cited. 